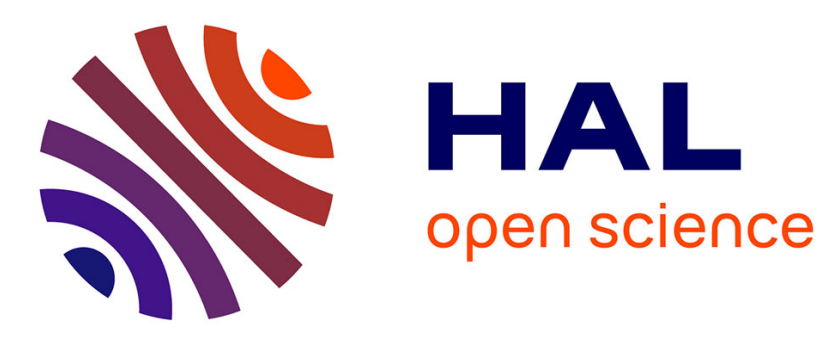

\title{
Un générateur d'impulsions de haute tension à débit élevé
}

\author{
G. Nenciarini, G. Kamarinos
}

\section{To cite this version:}

G. Nenciarini, G. Kamarinos. Un générateur d'impulsions de haute tension à débit élevé. Revue de Physique Appliquée, 1969, 4 (1), pp.94-96. 10.1051/rphysap:019690040109401 . jpa-00243162

\section{HAL Id: jpa-00243162 https://hal.science/jpa-00243162}

Submitted on 1 Jan 1969

HAL is a multi-disciplinary open access archive for the deposit and dissemination of scientific research documents, whether they are published or not. The documents may come from teaching and research institutions in France or abroad, or from public or private research centers.
L'archive ouverte pluridisciplinaire HAL, est destinée au dépôt et à la diffusion de documents scientifiques de niveau recherche, publiés ou non, émanant des établissements d'enseignement et de recherche français ou étrangers, des laboratoires publics ou privés. 


\title{
UN GÉNÉRATEUR D'IMPULSIONS DE HAUTE TENSION A DÉBIT ÉLEVÉ
}

\author{
Par G. NENGIARINI et G. KAMARINOS,
}

École Nationale Supérieure d’Électronique et de Radioélectricité de Grenoble, 23, rue des Martyrs, 38-Grenoble.

\author{
(Reçu le 30 janvier 1969.)
}

Résumé. - Le générateur fournit des impulsions rectangulaires de haute tension (jusqu’à $10 \mathrm{kV}$ ) et il est capable de débiter des courants de plusieurs ampères.

On peut faire varier, de façon continue, l'amplitude des impulsions $(0,5-10 \mathrm{kV})$, leur durée $(0,1-8 \mu \mathrm{s})$, et la fréquence de répétition $(0,1$ à $3 \mathrm{~Hz})$.

\begin{abstract}
The generator described gives H.T. rectangular pulses (up to $10 \mathrm{kV}$ ) with currents of many amperes.

It is possible to vary continuously the amplitude of the pulses $(0.5-10 \mathrm{kV})$, their width and their repetition frequency $(0.1-3 \mathrm{~Hz})$.
\end{abstract}

1. Introduction. - L'appareil a été réalisé pour certaines études sur les matériaux semiconducteurs (germanium notamment), lorsqu'ils sont soumis à des champs électriques élevés (quelques $\mathrm{kV} / \mathrm{cm}$ ).

Puisque la résistance des barreaux à étudier est relativement faible (quelques centaines d'ohms), la puissance requise est élevée. Pour éviter un échauffement notable du matériau, on ne peut lui appliquer des tensions continues permanentes; on doit donc recourir à un régime impulsif, laissant des temps morts assez longs.

Divers montages déjà réalisés $[1,2$ et 3$]$ ne conciliaient pas nos exigences en puissance (de l'ordre de quelques centaines de $\mathrm{kW})$, en durée $(0,1$ à $8 \mu \mathrm{s})$ et en amplitude de l'impulsion (jusqu'à $10 \mathrm{kV}$ ).

C'est ainsi que nous avons été amenés à réaliser [4] un générateur de puissance, fournissant des impulsions de haute tension, de forme rectangulaire, de faible durée et de fréquence de répétition basse. L'appareil peut néanmoins être utilisé pour d'autres recherches, pour lesquelles des caractéristiques semblables sont nécessaires (étude des ferrites à fort champ magnétique, comportement des décharges des gaz dans les tubes, etc.).

2. Principe du fonctionnement. - Une source de haute tension électrique alimente une cellule résistancecapacité; cette dernière joue le rôle d'un "réservoir d'énergie ». Par le moyen d'un système de commutation, la capacité se branche périodiquement à la charge, durant un laps de temps égal à la durée désirée de l'impulsion. 


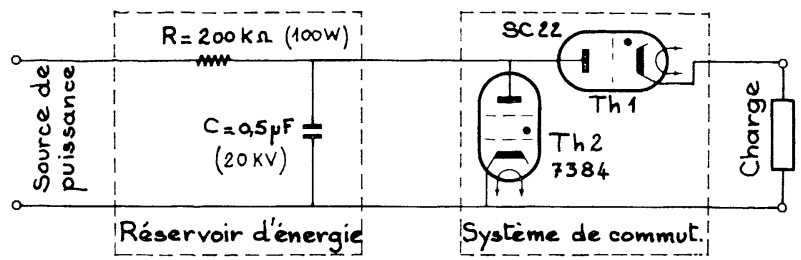

FIG. 1. - Principe de la chaîne de puissance.

Le système de commutation comprend deux thyratrons à hydrogène Th1 (SG 22) et Th2 (7384), choisis principalement pour leur grand pouvoir de coupure. De plus, leur faible temps de récupération (une dizaine de $\mu s$ ) permettrait de les employer à des fréquences de récurrence bien supérieures à celles que nous avons utilisées à cause de la nécessité d'évacuer l'énergie dissipée dans la charge d'épreuve.

3. Schéma d'ensemble de l'appareil. - Un générateur d'impulsions de tension, en forme de dents de

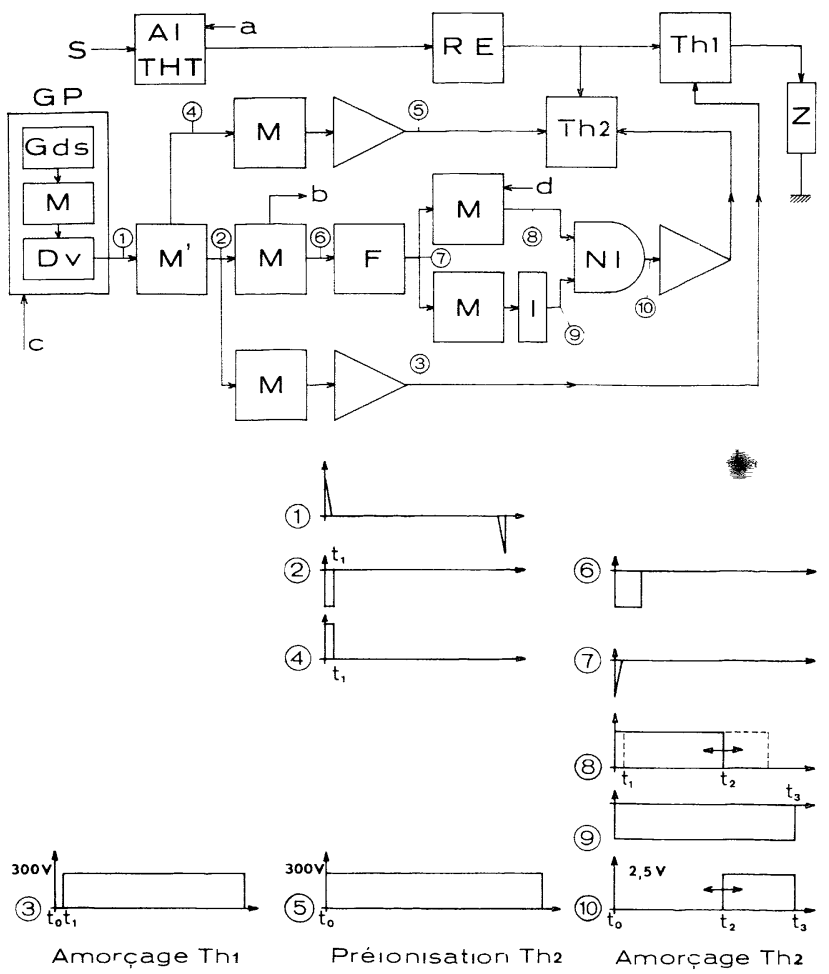

FiG. 2. - Schéma synoptique du générateur et formes des impulsions issues des différents circuits de commande : a, réglage de l'amplitude de l'impulsion; b, signal de synchronisation ; c, réglage de la fréquence de répétition; $d$, réglage de la durée de l'impulsion; $\mathrm{S}$, secteur ; Al THT, alimentation très haute tension; $\mathrm{RE}$, réservoir d'énergie; $Z$, charge; GP, générateur pilote; Gds, générateur des impulsions en forme de "dents de scie "; $M$, bascule monostable; $M^{\prime}$, décaleur (bascule monostable); Dv, dérivateur RC; $\mathrm{F}$, circuit de mise en forme ; I, inverseur ; NI, porte logique "NI ". scie et de fréquence égale à la fréquence de répétition désirée, suivi d'une bascule monostable et d'un circuit dérivateur RG, débite sur trois chaînes de circuits de pilotage; ces derniers sont destinés à fournir les signaux nécessaires à la commande des deux thyratrons.

Les impulsions issues des circuits de pilotage sont de faible amplitude $(2,5 \mathrm{~V})$; elles doivent être amplifiées (jusqu'à $300 \mathrm{~V}$ environ) afin de pouvoir attaquer les grilles des thyratrons. Nous avons utilisé, dans ce but, trois amplificateurs digitaux identiques.

Un schéma synoptique de l'ensemble du générateur (circuits de commande compris) est donné.

Une impulsion est d'abord appliquée à la grille 1 de Th2, au temps $t=t_{0}$, et sert à sa préionisation.

Au temps $t=t_{0}+0,6 \mu \mathrm{s}=t_{1}$, une autre impulsion est appliquée à la grille de Th1 qui s'amorce; ceci correspond au début de l'impulsion sur la charge.

Une troisième impulsion, décalée dans le temps par rapport aux deux premières, s'applique à la grille 2 de Th2 et l'amorce au temps $t=t_{2}$; ceci correspond à la fin de l'impulsion sur la charge qui reçoit ainsi une impulsion de durée $\tau=t_{2}-t_{1}\left(=t_{2}-t_{0}-0,6 \mu \mathrm{s}\right)$.

4. Performances et caractéristiques générales. - Le générateur délivre des impulsions de forme approximativement rectangulaire.

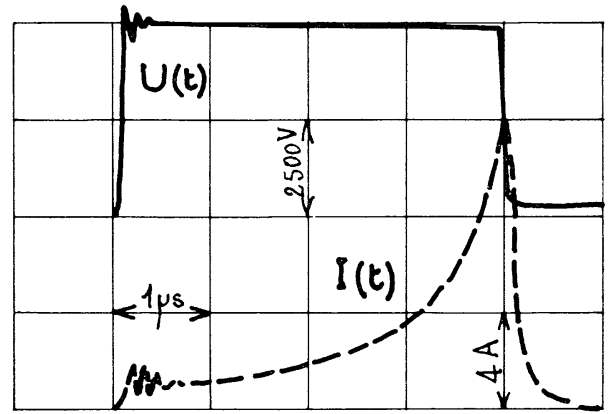

Fig. 3. - Impulsions de courant et de tension sur un barreau de germanium de type $\mathrm{N}$ de dimensions $1 \times 2 \times 20 \mathrm{~mm}$ et de résistivité $\rho=50 \Omega \mathrm{cm}$.

Temps de montée de l'impulsion : de l'ordre de $15 \mathrm{~ns}$; Temps de descente : du même ordre de grandeur;

Amplitude : de $500 \mathrm{~V}$ à $10 \mathrm{kV}$ (réglage continu);

Palier de l'impulsion: horizontal à $5 \%$ près (pour une impulsion de $4 \mu$ s de durée);

Durée de l'impulsion : réglable de façon continue entre 0,1 et $8 \mu$ s avec répartition en quatre gammes : $0,1-0,2 \mu \mathrm{s} ; 0,2-0,5 \mu \mathrm{s} ; 0,5-2 \mu \mathrm{s} ; 2-8 \mu \mathrm{s}$;

Fréquence de répétition: à réglage continu, de 0,1 à $3 \mathrm{~Hz}$ avec répartition en trois gammes : $0,1-0,3 \mathrm{~Hz}$; $0,3-1 \mathrm{~Hz} ; 1-3 \mathrm{~Hz}$;

Puissance consommée : $600 \mathrm{~W}$ environ;

Impédance de charge : supérieure à $100 \Omega$;

Puissance crête maximale: de l'ordre de $200 \mathrm{~kW}$;

Possibilités de modification des caractéristiques : la conception même des circuits permet de modifier la durée et la fréquence de répétition des impulsions. 
Cependant, l'augmentation de la durée au-delà d'une dizaine de $\mu$ s entraînerait une dégradation du palier de l'impulsion. On pourrait conserver la forme initiale de l'impulsion, en augmentant la valeur de la capacité $\mathrm{G}$, mais alors on devrait réduire la fréquence de répétition (limitation imposée par la puissance consommée sur la charge).

Si l'on veut augmenter la fréquence de répétition, quand la durée de l'impulsion est inférieure à $8 \mu \mathrm{s}$, on doit diminuer la valeur de la capacité $\mathrm{C}$, pour que celle-ci ait le temps de se charger entre deux impulsions.

Conclusion. - Ge qui distingue le générateur que nous avons décrit des autres réalisations est la simpli- cité et l'efficacité de la chaîne de puissance ainsi que la conception et la réalisation des circuits de commande (entièrement de composants solides); nous sommes ainsi arrivés à obtenir assez facilement des impulsions de puissance crête de l'ordre de $200 \mathrm{~kW}$ et de durée à réglage continu de 0,1 à 8 us.

Ce générateur est destiné à une série d'études sur le comportement des porteurs de charge sous grand champ électrique dans les semiconducteurs; en particulier, il nous a permis d'étudier, par le moyen de l'effet Hall, la répartition et le type de porteurs de charge dans un barreau de germanium, soumis à une impulsion de haute tension [5].

\section{BIBLIOGRAPHIE}

[1] Nacibov (A. S.) et Lomakin (V. L.), Generator for rectangular pulses, Inst. and experimental techniques, May-June 1965, no 3 , pp. 592-594.

[2] Char (T.) et GunN (M. W.), A high voltage pulse generator using switched thyratrons, Elect. Eng., June 1965, 37, no 448, 401-403.

[3] GRANGER (R.), Réalisation d'un générateur d'impulsion de grande puissance, $J$. Physique, octobre 1965, 26, $213 \mathrm{~A}-214 \mathrm{~A}$.
[4] E.N.S.E.G., Générateur d'impulsions rectangulaires de puissance, récurrentes, Projet d'Élèves (19671968).

[5] Kamarinos (G.), Nenciarini (G.), Boudouris (G.) et Buyle-Bodin (M.), C. R. Acad. Sc., 20 janvier 1969, série $B, 268,204-207$. 